\title{
Diagnostic Value of C-reactive Protein in Determining of Gestational Diabetes Mellitus (GDM)
}

\author{
Z. Ahi ${ }^{1}$, N. Kariman ${ }^{2 *}$, S.Zahedi Asl ${ }^{3}$ and N. Shakeri ${ }^{4}$ \\ ${ }^{1}$ Master of Midwifery,. Nursing and Midwifery School, Student research Office, \\ Shahid Beheshti University of Medical Sciences. \\ ${ }^{2} \mathrm{PhD}$ in Reproductive health.Assistant professor,. Midwifery Faculty,. Nursing \\ and Midwifery School,. Shahid Beheshti University of Medical Sciences . \\ ${ }^{3} \mathrm{PhD}$,. Professor of Endocrine Physiology,. Endocrine Research Center,. Research \\ Institute for Endocrine Sciences,. Shahid Beheshti University of Medical Sciences. \\ ${ }^{4} \mathrm{PhD}$ in Biostatistics, Medical Faculty, Paramedical School, \\ Shahid Beheshti University of Medical Sciences.
}

http://dx.doi.org/10.13005/bbra/2093

(Received: 02 April 2016; accepted: 14 May 2016)

This study was designed to evaluate diagnostic value of C-reactive protein in determining of gestational diabetes mellitus. The present case-control study was conducted on 60 pregnant women with GDM (case group) and 120 women with normal pregnancy (control group) referred to Ayatollah Taleghani and mahdiyeh Hospitals. The serum level of qualitative and quantitative CRP was measured and diagnostic value of CRP was determined. In this study information form was completed by interview and sampling was performed by convenience method. Data were analyzed by SPSS-17 and significance level of $p<0.05$ was considered. Serum CRP value in GDM and control groups was 3/ $59 \pm 3 / 2$ and $1 / 44 \pm 3 / 3 \mathrm{mg} /$ liter, respectively. Regarding to cut-off of $2 / 2 \mathrm{mg} / \mathrm{liter}$, sensitivity, specificity, positive predictive value and negative predictive value of quantitative CRP were $71 \%, 60 \%, 47 \%$ and $81 \%$, respectively in diagnosis of GDM. The under curve area was $0 / 70$. Sensitivity, specificity, positive predictive value and negative predictive value of qualitative CRP in diagnosis of GDM was calculated $33 / 3 \%, 98 / 3 \%, 90 \%$ and $74 \%$, respectively. It seems that measuring CRP in pregnant women with GDM risk factors can be used as a simple, new and reliable method to screen gestational diabetes mellitus.

Key words: Gestational Diabetes, C - reactive protein.

Diabetes is the most common medical condition during pregnancy, and its early detection is necessary to prevent prenatal and maternal complications ${ }^{1}$. Gestational diabetes is defined as carbohydrate intolerance, with its onset during pregnancy $[1,2,3$. In the USA, GDM affects up to $10 \%$ of all pregnancies and, $5-10 \%$ of women with GDM are found to have type 2 diabetes mellitus ${ }^{4,5,6,7}$. Approximately $50 \%$ of women diagnosed with this metabolic condition are expected to develop T2DM over $10-30$ years ${ }^{4}$.The

\footnotetext{
* To whom all correspondence should be addressed. Fax: +98(21)88202512

E-mail: n_kariman@yahoo.com,n_kariman@sbmu.ac.ir
}

prevalence of gestational diabetes in Iran was reported $4.8 \%{ }^{8}$.

Despite having adverse consequences on the health of mother and fetus, GDM remains a neglected maternal health issue ${ }^{9}$. Gestational diabetes, a health threatening factor for mother and fetus, is responsible for a broad range of prenatal and neonatal complications, including fetal macrosomia, birth trauma, preterm rupture of membranes, chorioamnionitis, neonatal hypoglycemia, preterm delivery, respiratory distress syndrome, neonatal hyperbilirubinemia, future obesity and diabetes, and also maternal complications such as increased prevalence of hypertension, and increased need for caesarian section delivery ${ }^{1,10-12}$. Gestational diabetes is 
associated with increased incidence of cardiovascular diseases and diabetes type II in future $^{1,3,7,11-13}$.

Like diabetes type II, gestational diabetes is induced by insulin resistance and impaired insulin discharge or both ${ }^{7,13}$. Insulin resistance (IR) is a metabolic state associated with increased risk of type 2 diabetes and cardiovascular disease through a variety of different molecular mechanisms. It is an inflammatory state characterized by elevated levels of cytokines such as high sensitivity C-reactive protein ${ }^{14}$. There is evidence that inflammation as a central feature in the development of Type 2 diabetes mellitus is associated with insulin resistance. Inflammation has been reported in GDM but the prognostic significance of this remains to be fully elucidated ${ }^{15}$, 16. Molecular basis of the relationship between infection and diabetes is associated with reaction of cytokines such as interleukin 6 and tumor necrosis factor (TNF). These inflammatory agents are insulin antagonists, which increase resistance to insulin and stimulate acute phase of inflammatory response $^{7,17}$. C-reactive protein (CRP) is an acute phase protein released by the liver after the onset of inflammation or tissue damage ${ }^{18-21}$. C-reactive protein is an acute-phase reactant that, at subclinical elevations, is a marker for endothelial damage, cardiovascular disease, and obesity ${ }^{22,23}$. Very small changes in CRP levels, detected with highly-sensitive assays, may also occur in association with metabolic stresses in the absence of acute or chronic inflammatory states as they have traditionally been viewed ${ }^{20}$. Recent studies have shown that people with increased C-reactive protein serum levels are exposed to risk of diabetes, hypertension, and cardiovascular diseases ${ }^{24-26}$. In pregnancy an increase in maternal CRP has been associated with a variety of pregnancy complications including early pregnancy loss, preterm delivery, preeclampsia, fetal growth restriction, premature rupture of the membranes and chorioamnionitis ${ }^{18}$.

There is uncertainty as to the optimal approach for screening and diagnosis of gestational diabetes mellitus (GDM). The U.S. Preventive Services Task Force concluded that there was insufficient evidence upon which to make a recommendation regarding routine screening of all pregnant women ${ }^{27}$. Every pregnant women should undergo screening for gestational diabetes between 24 and 28 gestational weeks, and women with risk factors of gestational diabetes such as history of gestational diabetes, diabetes, aged 25 years and older, BMI e” 30, polycystic ovary syndrome, use of corticosteroids, history of macrosomal neonate, polyhydramnios in current pregnancy should be screened for gestational diabetes in their first perinatal care visit at whatever gestational age $28,29,30$. To screen for gestational diabetes, glucose challenge test (GCT) is used with 50 grams of oral glucose as a standard test during 24 to 28 gestational weeks. Impaired GCT means $130 \mathrm{mg} / \mathrm{dl}$ or more, and is followed by glucose tolerance test (GTT) ${ }^{1}$. Although GCT with 50 grams oral glucose is considered a standard test of gestational diabetes, this test has many problems such as: it is time consuming and expensive, it shows different results in different ethnicities and races, and high amounts of sugar consumption can cause intolerance in some patients. Given these problems, and that existing gestational diabetes screening tests are time consuming and expensive, it seems cheaper tests with more sensitivity and specificity are required.

This study was conducted to determine C-reactive protein strength in detecting gestational diabetes in pregnant women presented to teaching medical centers affiliated to Shahid Beheshti University of Medical Sciences during 2011-2012.

\section{MATERIALSANDMETHODS}

The study protocol (No. 90-1-86-7944-1) was approved by the Ethics Committee of Shahid Beheshti Medical University. In this case-control study, 180 pregnant women attending perinatal clinic, obstetrics department, endocrinology department, and endocrine clinic at teaching hospitals affiliated to Shahid Beheshti University of Medical Sciences (Mahdieh and Taleghani hospitals) were selected. Of the eligible participants, 60 were with gestational diabetes (case group), and 120 without gestational diabetes (control group).

An information form was used for data collection purposes that included two sections; first section: study inclusion and exclusion criteria, and second section: demographic details (age, education, occupation, and socio-economic 
status), obstetric history (number of pregnancies, abortions, parity, and type of previous deliveries, gestational age, first trimester height and weight, weight at sampling), results checklist, qualitative serum CRP level, quantitative serum CRP level, glucose screening test during 24-28 weeks, and GTT if GCT shows high blood sugar, and reported results of oral and dental examinations by the dentist in their files. GCT and GTT test results in their files were used to detect gestational diabetes. In this study, GCT $>120 \mathrm{mg} / \mathrm{dl}$ was considered impaired. During gestational age 24-28 weeks glucose tolerance was tested by 75 grams of oral glucose, and fasting blood sugar was measured one and two hours after receiving glucose. Gestational diabetes was diagnosed if test results were impaired in at least one of the tests. Test results were described as fasting plasma glucose (FBS) $\geq 92 \mathrm{mg} / \mathrm{dl}$, blood sugar one hour after receiving glucose $\geq 180 \mathrm{mg} / \mathrm{dl}$, two hours after receiving glucose $\geq 153 \mathrm{mg} / \mathrm{dl}^{28,31}$.

All subjects were collected by an obstetrician. For scientific validation of CRP kit, CRP was measured using kits produced by Pars Azmon Company, and quantitative measurement of this marker was performed using auto-analyzer (Hitachi 902, Japan). Blood sugar was measured using glucose oxidase kits (Ideal Systems, Germany). Calibration method was used to ascertain reliability of kits. To determine serum CRP level, all tests were carried out at Shariat-Razavi Hospital laboratory by the same person, using the same device and the same method.

Content validity of data form and checklist were confirmed by faculty members of Shahid Beheshti University of Medical Sciences. Reliability of data form and checklist was determined using test-retest method (questions with $85 \%$ or more consistency were accepted). To decide reliability of test technician, concurrent observation method was used (KAPPA coefficient $0.9)$.

In this study, convenient sampling method was used, and in accordance with prevalence of gestational diabetes and errors á $=0.05$ and $\hat{a}=0.02$, sample size was found 60 women in gestational diabetes group and 120 women in control group. Study subjects were aged 18-35 years, with gestational age 24-28 weeks based on the exact first day of their last menstruation or, their first trimester ultrasound result. Women with following conditions were excluded: diabetes in the first trimester of pregnancy, or before pregnancy or in previous pregnancies, known diseases, hypertension or preeclampsia in the current pregnancy and active infection (purulent, yellow, and smelly discharge, burning, itching, abdominal pain, fever, chills, and tooth infection) at the time of sampling, women with history of birth of neonates over 4000 grams, history of preeclampsia, or eclampsia or hypertension, or history of stillbirth, history of neonatal or fetal abnormality, history of diabetes and multiple abortions in previous pregnancies, history of smoking, alcohol and substance use before and during pregnancy, family history of diabetes type II in the first degree relative, history of seasonal allergies, history of polycystic ovary syndrome and hyperlipidemia, use of non-routine medication during pregnancy, use of antibiotics 2 weeks before sampling, and glucosuria in the first perinatal visit.

After explaining objectives and nature of the study, and obtaining informed consents of women that met study inclusion criteria (through completing the first section of data form), the second section was completed through interviews with 60 pregnant women with definite diagnosis of gestational diabetes (as the case group) and 120 pregnant women without gestational diabetes (as the control group). Blood samples were taken from subjects and centrifuged to determine serum CRP level. Qualitative and quantitative serum CRP levels were determined 48-72 hours after sampling at Shariat-Razavi Hospital laboratory by a laboratory technician. Qualitative measurement of CRP was performed using latex agglutination method with threshold of $6 \mathrm{mg} / \mathrm{l}$ reported as plus, and quantitative measurement of CRP was conducted using immunoturbidometric method with CRP kits with $0.02-40 \mathrm{mg} / \mathrm{l}$ threshold.

In this study, statistical software SPSS16 was used for analysis of data. Confidence interval was considered 95\%. All collected data were presented as charts and tables of frequency distribution, quantity and percentage. Descriptive statistics were used in tabulation of frequency. To assess differences between case and control groups, independent t-test was used for quantitative variables and chi-square for qualitative variables, and Mann-Whitney for ranked variables. 
ROC curve was used to determine sensitivity and specificity of quantitative CRP test and quantitative serum CRP level threshold.

\section{RESULTS}

In this study, sampling was carried out during June-December 2011, and 180 subjects (60 as the case and 120 as the control groups), with similar demographic and obstetrics characteristics in both groups were selected. Statistical tests revealed insignificant differences between the two groups in terms of age, number of pregnancies, first trimester BMI, and oral and dental condition.

Most subjects in the case and the control groups were high school graduates $(46.7 \%$ and $52.5 \%$ respectively), and the majority in the control (98.3\%) and the case $(96.7 \%)$ groups were housewives. Most subjects in the control (55.8\%) and the case (45\%) groups were nulliparous, and the majority in the control (64.2\%) and the case (55\%) were primiparous with no previous births at all. There were insignificant differences in demographic details between the two groups. Table 1 presents confounding variables of age, number of pregnancies, and dental health indicator (DMF) in both groups. Statistical tests showed similarity between groups in terms of the above variables.

Table 1. Frequency of women with and without gestational diabetes according to demographic and obstetrics characteristics

\begin{tabular}{lccc}
\hline & Women with GDM (case) & Women without GDM (control) & P-value \\
\hline Age (years) & $27.28(4.6)$ & $26.81(3.8)$ & $0.49^{*}$ \\
Number of parities & $0.68(0.91)$ & $0.51(0.79)$ & $0.19^{* *}$ \\
$1^{\text {st }}$ trimester BMI $\left(\mathrm{kg} / \mathrm{m}^{2}\right)$ & $24.68(3.2)$ & $23.98(3)$ & $0.15^{*}$ \\
Dental health index (DMF) & $5.43(2.06)$ & $5.12(2.63)$ & $0.43^{*}$ \\
\hline
\end{tabular}

Used tests to analysis of variables: * Independent T-test, ** U Mann-whitney test

Table 2. Sensitivity and specificity of serum CRP level test for values of different cuts in study subjects

\begin{tabular}{lcc}
\hline Quantitative CRP & Sensitivity & Specificity \\
\hline-.9000 & 1.000 & 1.000 \\
.1500 & 1.000 & .958 \\
1.8500 & .717 & .433 \\
1.9500 & .717 & .417 \\
2.2000 & .717 & .392 \\
2.5000 & .683 & .392 \\
2.6500 & .683 & .358 \\
23.4500 & .017 & .000 \\
27.0000 & .000 & .000 \\
\hline
\end{tabular}

Qualitative serum CRP level was negative in the majority of study subjects in the case group (66.7\%) and the control group (98.3\%). Fewest subjects in the case and the control groups (1.7\%) had qualitative CRP levels 3+ and 1+, respectively. Mann-Whitney test results revealed significant differences in qualitative serum CRP between the two groups $(\mathrm{P}<0.0005)$. Sensitivity, specificity, positive and negative predicting values of qualitative CRP test to predict gestational diabetes were estimated at 33.3\%, 98.3\%, 90\%, and 74\%, respectively.

Table 3. Frequency of quantitative serum CRP according to threshold (cut-off point) of $2.2 \mathrm{mg} / \mathrm{l}$ in the two groups

\begin{tabular}{lccccc}
\hline \multirow{2}{*}{$\begin{array}{l}\text { Quantitative } \\
\text { CRP }\end{array}$} & \multicolumn{2}{c}{$\begin{array}{c}\text { Women with gestational diabetes } \\
\text { (Case group) }\end{array}$} & & \multicolumn{2}{c}{$\begin{array}{c}\text { Healthily pregnant women } \\
\text { (Control group) }\end{array}$} \\
\cline { 2 - 3 } \cline { 5 - 6 } & Frequency & Percentage & & Frequency & Percentage \\
\hline & 17 & & & 73 & 60.8 \\
$>2.2$ & 43 & 71.7 & & 47 & 39.2 \\
Total & 60 & 100 & & 120 & 100 \\
\hline
\end{tabular}


The majority of study subjects in the case group (25\%) had quantitative serum CRP level a little over 10, and in the control group less than 1 $\mathrm{mg} / \mathrm{l}$. Independent t-test showed significant differences in quantitative serum CRP level between the two groups $(\mathrm{P}<0.0005)$. Standard deviation of quantitative CRP in the case group was estimated at 3.59 (3.22) and in the control group 1.44 (3.32) mg/l. There were significant correlations between blood sugar level in glucose screening test with quantitative CRP and qualitative CRP, and between quantitative and qualitative CRP levels $(\mathrm{P}<0.0005)$.

Given the non-normal distribution of quantitative CRP according to KolmogorovSmirnov test, geometric mean was calculated. Using ROC curve (figure 1 ) and minimum and maximum values of quantitative CRP threshold (table 2), appropriate quantitative CRP threshold for diagnosing gestational diabetes was found $2.2 \mathrm{mg} /$ 1 . The area under the curve was found 0.7 , indicating acceptability of quantitative CRP test to predict gestational diabetes.

According to $2.2 \mathrm{mg} / \mathrm{l}$ threshold found using ROC curve, 43 women in the case group (71.7\%) had positive results and 17 (28.3\%) had negative results in quantitative serum CRP test.

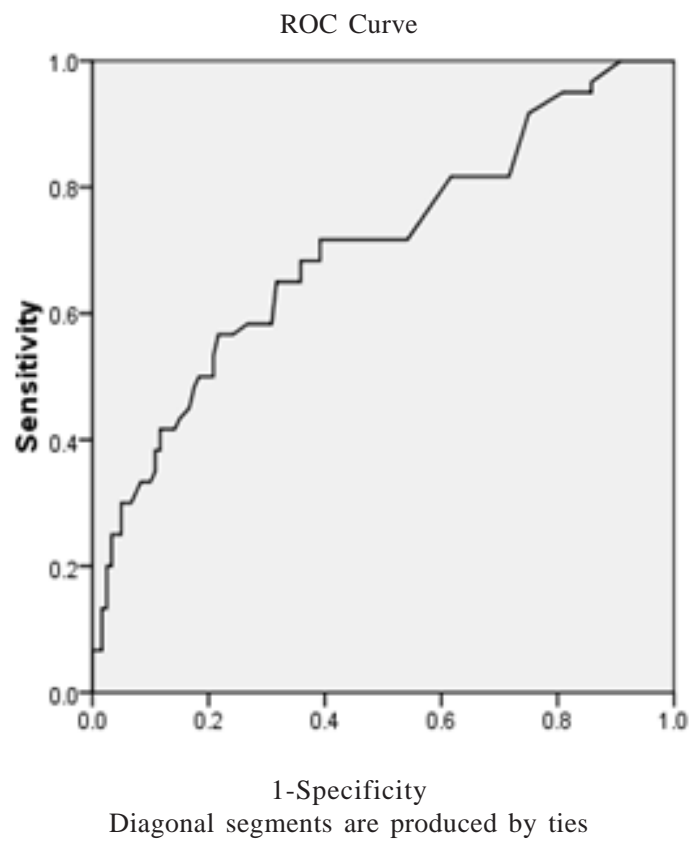

Fig. 1. Quantitative serum CRP curve for predicting gestational diabetes in study subjects
Also, in the control group, 73 women (60.8\%) were negative and 47 (39.2\%) were positive in the above test (table 3).

According to results in the above table, sensitivity, specificity, and positive and negative predicting value of quantitative serum CRP test with $2.2 \mathrm{mg} / \mathrm{l}$ threshold were found $71 \%, 60 \%, 47 \%$, and $81 \%$, respectively.

\section{DISCUSSION}

The present study revealed significant differences in qualitative and quantitative serum CRP levels between the case and the control groups $(\mathrm{P}<0.0005)$, and that diagnostics strength of quantitative serum CRP was acceptable.

Despite 4 decades of research, a general consensus has not been reached about optimal approach to screening for gestational diabetes. The important challenge is in the choice between general or elective screening, and also diagnostic threshold of GCT [1]. Cunningham et al. (2010) reported sensitivity and specificity of GCT test in screening for glucose with $140 \mathrm{mg} / \mathrm{dl}$ threshold were: $80 \%$ and $82-86 \%$ respectively, and with 130 $\mathrm{mg} / \mathrm{dl}$ threshold, $90 \%$ and $75-80 \%$, respectively. In a study by Pontamanopol \& Takasacal (2008) [32], sensitivity and specificity of GCT test in predicting gestational diabetes were found $60.8 \%$ and $75 \%$, respectively, by determining threshold of $177 \mathrm{mg} /$ dl. These indices are intensely controlled by gestational diabetes risk factors, which highlight the issue of elective screening. Furthermore, due to different values of diagnostic glucose plasma threshold found in different studies, sensitivity and specificity of GCT test is still controversial among researchers.

In several recent studies, fasting plasma glucose (FPG) has been examined as a glucose screening method. Sensitivity and specificity of FPG test in diagnosing gestational diabetes were found $63.89 \%$ and $76.56 \%$ by Mirfazi et al. (2011), $80 \%$ and $92 \%$ by Kashi et al. (2007), $86 \%$ and $100 \%$ by Agarwal et al. (2009), and $80 \%$ and $40 \%$ by Aguire et al. (2009), respectively [33, 34, 35, and 36]. Also, area under FPG curve in diagnosing gestational diabetes in studies by Mirfazi et al. (2011), Kashi et al. (2007), and Agarwal et al. (2009) was found 0.75, 0.85 , and 0.83 , respectively. These results were found in studies on subjects at risk of gestational 
diabetes, which shows acceptability of FPG test in diagnosing gestational diabetes in high-risk populations. In the present study, subjects had none of the gestational diabetes risk factors, which is indicative of higher strength of present study results.

In a study by Syngelaki et al was reported in pregnancies that develop GDM there is no evidence of an inflammatory response at 11-13 weeks' gestation and the levels of serum TNF-á and Hs-CRP are not useful in first-trimester screening for GDM [15]. In the other study by Corcoran et al however, higher levels of CRP were found to be significantly associated with macrosomia but hS-CRP did not demonstrate additional predictive ability for GDM [37]. In the present study, sensitivity and specificity of CRP test were found $33.3 \%$ and $98.3 \%$, respectively, which shows that, despite acceptable attributes, qualitative CRP test has unacceptable sensitivity in predicting gestational diabetes. Positive and negative predicting values of qualitative CRP test in predicting gestational diabetes were estimated at $90 \%$ and $74 \%$, respectively. Moreover, in this study, sensitivity and specificity of positive and negative predicting value of quantitative CRP test with $2.2 \mathrm{mg} / \mathrm{l}$ threshold to predict gestational diabetes were $71 \%, 60 \%, 47 \%$ and $81 \%$, respectively, without considering any gestational diabetes risk factors, which is indicative of acceptable diagnostic strength. Area under the curve was found 0.7 . If gestational diabetes risk factors such as obesity, age, multiparity, history of gestational diabetes, and relevant obstetric history exist, diagnostic strength of this test in diagnosing gestational diabetes will increase, which requires further and more comprehensive and cohort studies.

Given the results obtained and comparison of the two assessment methods of qualitative serum CRP using CRP kits and latex agglutination method at threshold of $6 \mathrm{mg} / \mathrm{l}$, and quantitative serum CRP test using immunoturbidometric method at threshold of 2.2 $\mathrm{mg} / \mathrm{l}$, it was shown that using CRP kits had sensitivity of $33.3 \%$ and specificity of $98.3 \%$. In this study, sensitivity and specificity of quantitative serum CRP test was found acceptable (71\% and 60\%, respectively), and area under curve was found 0.7 , which indicates that quantitative
CRP test is acceptable in predicting gestational diabetes.

Finally, following further and more comprehensive studies to assess diagnostic strength of serum CRP in pregnant women at risk of gestational diabetes, use of CRP test can be considered in pregnant women qualified for gestational diabetes risk factors, as a new, fast and reliable screening test.

\section{ACKNOWLEDGEMENTS}

This article was extracted from a thesis in master degree in midwifery at Shahid Beheshti University of Medical Sciences. All pregnant women admitted to teaching medical hospitals affiliated to Shahid Behashti University of Medical Sciences are hereby thanked for their cooperation in all stages of the study. Professors and Dean of Obstetrics Department of School of Midwifery and Nursing of Shahid Beheshti University of Medical Sciences are also appreciated for recognizing the need to conduct this study.

\section{REFERENCES}

1. Cunningham FG., et al., "Williams Obstetrics". 24th edition. New York, wendel T. 2014; 1125 , 1136, 1137, 1138, 1139,1140.

2. Ashwal, E. and M. Hod, "Gestational diabetes mellitus: Where are we now?" Clinica Chimica Acta. 2015; 7;451(Pt A):14-20. doi: 10.1016/ j.cca .2015 .01 .021$.

3. Horvath, K., et al., "Effects of treatment in women with gestational diabetes mellitus: systematic review and meta-analysis.” Bmj340: c1395; 2010. http://dx.doi.org/10.1136/ bmj.c1395

4. Monteiro, L. J., et al., "Fetal programming and gestational diabetes mellitus.”Placenta, 2015.pii: S0143-4004(15)30096-5. doi: 10.1016/ j.placenta.2015.11.015

5. Baraban, E., et al., "Peer Reviewed: Increasing Prevalence of Gestational Diabetes and Pregnancy-Related Hypertension in Los Angeles County, California, 1991-2003.” Preventing chronic disease 2008; 5(3). PMCID: PMC2483543

6. Li, X. and X. Lu, “Study on correlation between C-reactive protein and gestational diabetes mellitus.” Journal of Nanjing Medical University 2007; 21(6): 382-385. doi:10.1016/S10074376(07)60083-5 
7. Wolf, M., et al., First-trimester C-reactive protein and subsequent gestational diabetes. Diabetes care 2003; 26(3): 819-824. PMID:12610043

8. Keshavarz, M., et al., "Gestational diabetes in Iran: incidence, risk factors and pregnancy outcomes." Diabetes research and clinical practice 2005; 69(3): 279-286. PMID:16098925

9. Mohan, M. A. and A. Chandrakumar Evaluation of prevalence and risk factors of gestational diabetes in a tertiary care hospital in Kerala.”Diabetes \& Metabolic Syndrome: Clinical Research \& Reviews 2015. doi: 10.1016/ j.dsx.2015.09.002

10. Srichumchit, S., et al., Outcomes of pregnancy with gestational diabetes mellitus.International Journal of Gynecology \& Obstetrics 131(3): 251254. doi: 10.1016/j.ijgo .2015.05.033

11. Retnakaran, R. and B. R. Shah. "Mild glucose intolerance in pregnancy and risk of cardiovascular disease: a population-based cohort study." Canadian Medical Association Journal 2009; 181(6-7): 371-376. doi: 10.1503/ cmaj.090569

12. Donovan, P. J. and H. D. McIntyre. Drugs for gestational diabetes.”AustPrescr 2010; 33: 141144. http://dx.doi.org/10.18773/austprescr. 2010. 066

13. Feig, D. S., et al., "Risk of development of diabetes mellitus after diagnosis of gestational diabetes."Canadian Medical Association Journal 2008; 179(3): 229-234. doi:10.1503/cmaj.080012

14. O'Sullivan, E. P., et al., "A comparison of osteoprotegerin with adiponectin and highsensitivity C-reactive protein (hsCRP) as a marker for insulin resistance."Metabolism 2013; 62(1): 34-38. doi: 10.1016/j.metabol .2012.06 .005

15. Syngelaki, A., et al., "First trimester screening for gestational diabetes mellitus by maternal factors and markers of inflammation. Metabolism 2016; 65(3): 131-137. http://dx.doi.org/10.1016/ j.metabol.2015.10.029

16. Retnakaran, R., et al., "C-reactive protein and gestational diabetes: the central role of maternal obesity.” The Journal of Clinical Endocrinology \& Metabolism 2003; 88(8): 3507-3512. PMID:12915627

17. Dasanayake, A., et al. "Periodontal pathogens and gestational diabetes mellitus.” Journal of dental research 2008; 87(4): 328-333. PMCID: PMC2561333

18. Maguire, P. J., et al., "Maternal C-reactive protein in early pregnancy.”European Journal of Obstetrics \& Gynecology and Reproductive Biology 2015; 193: 79-82. doi: 10.1016/ j.ejogrb.2015.07.005

19. Pakzad, P. "Principles and interpretation of clinical serology experiments”. 11th edition. Noore danesh press. 2004; 169, 170, 171.

20. Kushner, I., “Acute phase reactants” UpToDate, Furst, D. E \& Romain, P. L (Eds.), 2015.

21. Morrow, D. A., Screening for cardiovascular risk with c-reactive protein.”UpToDate, Kaski, J \& Downey B (Eds.), Waltham, MA, 2015.

22. Berggren, E. K., et al., "First-trimester maternal serum C-reactive protein as a predictor of thirdtrimester impaired glucose tolerance." Reproductive Sciences: 2014; 22(1):90-3. doi: 10.1177/1933719114532843

23. Dehghani Firousabadi, R., "The Role of Metformin in Reduction SerumC-Reactive Protein Levels in Women with Polycystic Ovary Syndrome”.Int J Fertil Steril: 2010; 2008-0778.

24. Pradhan, A. D., et al.. "C-reactive protein, interleukin 6, and risk of developing type 2 diabetes mellitus.” Jama 2001; 286(3): 327-334. PMID:11466099

25. Dehghan, A., et al., "Genetic variation, Creactive protein levels, and incidence of diabetes.” Diabetes 2007; 56(3): 872-878. PMID:17327459

26. Lopez-Garcia, E., et al., "Consumption of trans fatty acids is related to plasma biomarkers of inflammation and endothelial dysfunction." The Journal of nutrition 2005; 135(3): 562-566. PMID:15735094

27. Hartling, L., et al., "Screening and diagnosing gestational diabetes mellitus.”Report No.: 12(13)-E021-EF, 2012

28. Thompson, D., et al., "Diabetes and Pregnancy." Canadian Diabetes Association 2013; 36. http:/ /guidlines.diabetes.ca

29. Custan ,D. A. and Jovanovic, L., “ Diabetes mellitus in pregnancy: Screening and diagnosis”. UpToDate, Nathan , D. M \& Greene, M. F (Eds.), 2015.

30. Jovanovic, L . "Patient information: Gestational diabetes mellitus (Beyond the the Basics) “. UpToDate, Nathan, D. M \&Greene, M. F (Eds.), 2015.

31. Custan ,D. A. and Jovanovic, L. “ Treatment and course gestational diabetes mellitus". UpToDate, Nathan, D. M \& Greene, M. F (Eds.), 85.133.137.136-7CBA809361; 2013.

32. Punthumapol, C. and P. Tekasakul. "50 Grams Glucose Challenge Test for Screening of Gestational Diabetes Mellitus in Each Trimester in Potential Diabetic Pregnancy.” Medical Journal of the Medical Association of Thailand 2008; 91(6): 787. PMID:18697374

33. Mirfeizi, M., et al., Examining diagnostic value 
of the fasting plasma glucose in screening gestational diabetes." Journal of Diabetes \& Metabolic Disorders 2011; 10(1): 12-.

34. Kashi, Z., et al. "Diagnostic value of fasting plasma glucose in screening of gestational diabetes mellitus.”International Journal of Endocrinology and Metabolism 2007(1, Winter): 1-4.

35. Agarwal, M., et al. "Gestational diabetes: fasting capillary glucose as a screening test in a multi ethnic, high risk population.” Diabetic Medicine 2009; 26(8): 760-765. doi: 10.1111/j.14645491.2009.02765.x
36. deAguiar, L. G. K., et al.. "Could fasting plasma glucose be used for screening high-risk outpatients for gestational diabetes mellitus?" Diabetes care 2001; 24(5): 954-955. PMID:11347763

37. Corcoran, S., et al., "739: Can first trimester high-sensitivity C-reactive protein and Sex Hormone Binding Globulin predict the onset of gestational diabetes? A prospective study of a high risk cohort.”American Journal of Obstetrics \& Gynecology 2016; 214(1): S388. doi: http:// dx.doi.org/10.1016/j.ajog.2015.10.787 